\title{
Rhenium reactivity - manipulation by ligand development
}

Alice Brink $^{a}$, Hendrik G. Visser ${ }^{a}$, Andreas Roodt ${ }^{a}$

${ }^{a}$ Department of Chemistry, University of the Free State, Nelson Mandela Drive, Bloemfontein, 9301, South Africa

Drug development can be approached from numerous angles, i.e. bio-activity, receptor binding, structural analysis combining small molecules with proteins, fragment-based drug design, functionalization of model pharmaceuticals, etc. Our interest is in the coordination of biomolecules and bifunctional chelators to ${ }^{99 \mathrm{~m}}$ technetium(I) and ${ }^{186 / 188}$ rhenium(I) for use as diagnostic or therapeutic radiopharmaceutical agents. The synthesis of radiopharmaceuticals can be accomplished by a good understanding of the transition metal chemistry of the specific reagents involved, but the kinetic effects caused by the bound molecules on the metal centre is critical for understanding reactivity, stability and structure. This study focuses on the kinetic and crystallographic properties of the fac-[M(L, $\left.\left.\mathrm{L}^{\prime}\right)(\mathrm{CO})_{3}(\mathrm{~S})\right](\mathrm{M}=\mathrm{Re}, \mathrm{Tc})$ model radiopharmaceutical complexes utilising the $\{2+1\}$ approach. The significant labilisation caused by the coordinated bidentate ligands and the isostructural behaviour of the crystallised complexes will be highlighted.

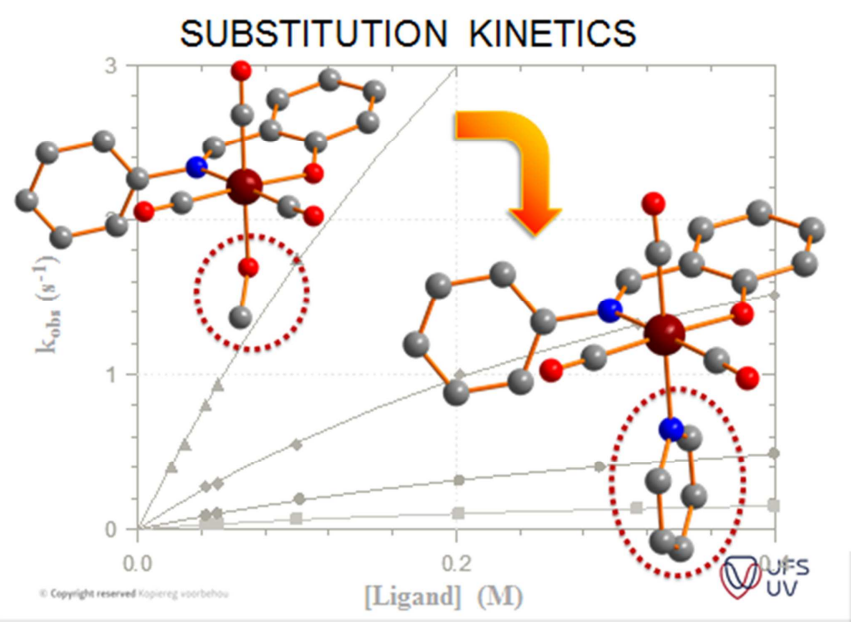

